PROCEEDINGS OF THE

AMERICAN MATHEMATICAL SOCIETY

Volume 138, Number 10, October 2010, Pages 3455-3465

S 0002-9939(10)10372-4

Article electronically published on May 5, 2010

\title{
EULER CHARACTERISTICS, AKASHI SERIES AND COMPACT $p$-ADIC LIE GROUPS
}

\author{
SIMON WADSLEY
}

(Communicated by Birge Huisgen-Zimmermann)

\begin{abstract}
We discuss Euler characteristics for finitely generated modules over Iwasawa algebras. We show that the Euler characteristic of a module is well-defined whenever the 0th homology group is finite if and only if the relevant compact $p$-adic Lie group is finite-by-nilpotent and that in this case all pseudo-null modules have trivial Euler characteristic. We also prove some other results relating to the triviality of Euler characteristics for pseudo-null modules as well as some analogous results for the Akashi series of Coates et al.
\end{abstract}

\section{INTRODUCTION}

In this paper, following 6, 9] we are interested in computing an Euler characteristic for finitely generated modules over Iwasawa algebras. Suppose that $G$ is a compact $p$-adic Lie group without $p$-torsion and $M$ is a finitely generated $\mathbb{Z}_{p} G$ module. If the homology groups $H_{i}(G, M)$ are finite for all $i$, then we say that the Euler characteristic is well-defined and takes the value

$$
\chi(G, M):=\prod_{i \geqslant 0}\left|H_{i}(G, M)\right|^{(-1)^{i}} .
$$

The following result is classical:

Theorem. Suppose that $G \cong \mathbb{Z}_{p}^{d}$.

(1) The Euler characteristic of a finitely generated $\mathbb{Z}_{p} G$-module is well-defined if and only if $H_{0}(G, M)$ is finite.

(2) Moreover, $\chi(G, M)=1$ whenever $M$ is a pseudo-null module with welldefined Euler characteristic.

Both parts of this theorem are known not to be true for general compact $p$-adic Lie groups $G$ without $p$-torsion; see $[8]$.

In this paper we prove that (1) holds if and only if $G$ is finite-by-nilpotent. Both directions depend on the key fact that the set $S:=\mathbb{Z}_{p} G \backslash \operatorname{ker}\left(\mathbb{Z}_{p} G \rightarrow \mathbb{Z}_{p}\right)$ is an Ore set in $\mathbb{Z}_{p} G$ precisely when $G$ is finite-by-nilpotent.

In addition, we prove that part (2) of the theorem holds whenever $G$ is finiteby-nilpotent. To prove this, we prove analogous results for the Akashi series of 8 and 7 . (see (2.8) for the definition). In particular we show that if $G \cong H \rtimes \Gamma$, then every finitely generated $\mathbb{Z}_{p} G$-module $M$ such that $H_{0}(H, M)$ is $\mathbb{Z}_{p} \Gamma$-torsion has

Received by the editors December 3, 2009, and, in revised form, January 5, 2010.

2010 Mathematics Subject Classification. Primary 11R23, 16U20.

Key words and phrases. Iwasawa algebra, Euler characteristic, Akashi series.

(C)2010 American Mathematical Society Reverts to public domain 28 years from publication 
well-defined Akashi series if and only if $H$ is finite-by-nilpotent, and if $G$ is finiteby-nilpotent, then such a module that is also pseudo-null has trivial Akashi series. This time the first part depends on the fact that $T:=\mathbb{Z}_{p} G \backslash \operatorname{ker}\left(\mathbb{Z}_{p} G \rightarrow \mathbb{Z}_{p} \Gamma\right)$ is an Ore set in $\mathbb{Z}_{p} G$ precisely when $H$ is finite-by-nilpotent. We prove the second part using the Hochschild-Serre spectral sequence. We could prove part (2) of the Euler characteristics result directly using the same techniques, but we do not because it follows immediately from the Akashi series result.

Whenever $M$ is finitely generated and torsion for a subset of $S$ (resp. $T$ ) that is an Ore set of $\mathbb{Z}_{p} G$, the Euler characteristic (resp. Akashi series) of $M$ is welldefined. This raises the interesting algebraic question of what the maximal Ore subsets of $S$ and $T$ are for general compact $p$-adic Lie groups $G$ (with $G \cong H \rtimes \Gamma$ for the $T$ case). Since the long exact sequence of $K$-theory used to formulate the main conjecture in [7] is defined for any such Ore set and the connecting map will remain surjective whenever $G$ is pro- $p$, this question may well also have arithmetic implications as the $\mathcal{M}_{H}(G)$-conjecture ([7, Conjecture 5.1]) could then be weakened without losing the means of defining a characteristic element that should be related to a $p$-adic L-function.

Following work of Serre 13 in the case where $M$ is finite, Ardakov and the author [5] have described the Euler characteristic of any finitely generated $p$-torsion module in terms of a notion of graded Brauer character for $M$ that is supported on the $p$ regular elements of $G$. It followed from this description that if $d_{G}(M)<\operatorname{dim} C_{G}(g)$ for every $p$-regular element of $G$ then $\chi(G, M)$ must be 1 . Here $d_{G}(M)$ denotes the canonical dimension of $M$ as defined in section 2.4 and $C_{G}(g)$ denotes the centraliser of $g$ in $G$.

Totaro had already extended Serre's work in a different direction. Instead of concentrating on $p$-torsion modules he computed Euler characteristics of modules that are finitely generated as $\mathbb{Z}_{p}$-modules. Part of his main result was the following:

Theorem (14, Theorem 0.1). Let $p$ be any prime number. Let $G$ be a compact $p$ adic Lie group of dimension at least 2 , and let $M$ be a finitely generated $\mathbb{Z}_{p}$-module with $G$-action. Suppose that the homology of the Lie algebra $\mathfrak{g}_{\mathbb{Q}_{p}}$ of $G$ acting on $M \otimes \mathbb{Q}_{p}$ is 0 ; this is equivalent to assuming that the homology of any sufficiently small open subgroup $G_{0}$ acting on $M$ is finite, so that the Euler characteristic $\chi\left(G_{0}, M\right)$ is defined. Then the Euler characteristics $\chi\left(G_{0}, M\right)$ are the same for all sufficiently small open subgroups $G_{0}$ of $G$.

The common value of these Euler characteristics is 1 if every element of the Lie algebra $\mathfrak{g}_{\mathbb{Q}_{p}}$ has centraliser of dimension at least 2 . Otherwise, there is an element of $\mathfrak{g}_{\mathbb{Q}_{p}}$ whose centraliser has dimension 1 , and then the common value is not 1 for some choice of module $M$.

Looking at these results together, and recalling that $d_{G}(M) \leq 1$ for any finitely generated $\mathbb{Z}_{p}$-module, we might be tempted to make the following conjecture:

Conjecture. If $G$ is a compact p-adic Lie group without p-torsion and $M$ is a finitely generated $\mathbb{Z}_{p} G$-module with well-defined Euler characteristic such that $d_{G}(M)<\operatorname{dim} C_{G}(g)$ for all $g \in G$, then $\chi(G, M)=1$.

In fact the results of 4.2 might indicate that an even stronger conjecture is true. Recall that every compact $p$-adic Lie group has an associated $\mathbb{Z}_{p}$-Lie algebra $\mathfrak{g}$ and that each automorphism of $G$ induces an automorphism of $\mathfrak{g}$. In this way, for each $g \in G$ conjugation by $g$ induces an element $\theta(g)$ of $G L(\mathfrak{g})$. Let $\mathfrak{g}^{0}(g)$ denote the 
generalised eigenspace $\theta(g)$ for the eigenvalue 1 ; that is,

$$
\mathfrak{g}^{0}(g):=\left\{x \in \mathfrak{g} \mid(\theta(g)-1)^{n}=0 \text { for some } n>0\right\} .
$$

Conjecture. If $G$ is a compact p-adic Lie group without p-torsion and $M$ is a finitely generated $\mathbb{Z}_{p} G$-module with well-defined Euler characteristic such that $d_{G}(M)<\operatorname{dim} \mathfrak{g}^{0}(g)$ for each $g \in G$, then $\chi(G, M)=1$.

Of course, if either $g$ has finite order or $g$ has infinite order and $\operatorname{dim} C_{\mathfrak{g}}(g)=1$, then $\mathfrak{g}^{0}(g)=C_{\mathfrak{g}}(g)$. Moreover, if $G$ is finite-by-nilpotent, then $\operatorname{dim} \mathfrak{g}^{0}(g)=\operatorname{dim} G$ for every $g \in G$.

\section{Preliminaries}

2.1. Notation. Let $G$ be a compact $p$-adic Lie group. We define the Iwasawa algebra

$$
\mathbb{Z}_{p} G:=\lim _{\longleftarrow} \mathbb{Z}_{p}[G / N]
$$

where $N$ runs over all the open normal subgroups of $G$ and $\mathbb{Z}_{p}[G / N]$ denotes the usual algebraic group algebra. If $H$ is a closed normal subgroup of $G$, we write $I_{H, G}$ for the kernel of the augmentation map $\mathbb{Z}_{p} G \rightarrow \mathbb{Z}_{p} G / H$.

Given a profinite ring $R$, we write $\operatorname{Pmod}(R)$ for the category of profinite left $R$-modules and continuous $R$-module homomorphisms. Then $H_{i}(G,-)$ is the $i$ th derived functor of the functor

$$
(-)_{G}: \operatorname{Pmod}\left(\mathbb{Z}_{p} G\right) \rightarrow \operatorname{Pmod}\left(\mathbb{Z}_{p}\right)
$$

that sends a module $M$ to its $G$-coinvariants $M / I_{G, G} M$. Since $(-)_{G}=\mathbb{Z}_{p} \otimes_{\mathbb{Z}_{p} G}(-)$ as functors, it follows that $H_{i}(G, M) \cong \operatorname{Tor}_{i}^{\mathbb{Z}_{p} G}\left(\mathbb{Z}_{p}, M\right)$ for each $i \geqslant 0$ (see [11, section 6.3] for more details).

If $X$ is a subset of a profinite group, we will write $\langle X\rangle$ for the closed subgroup of $G$ generated by $X$. We write $Z(G)$ for the centre of $G$.

If $S$ is a (left and right) Ore set in a ring $R$ and $M$ is an $R$-module, we write $R_{S}$ for the localisation of $R$ at $S$ and $M_{S}$ for the localisation of $M$ at $S$.

$\Gamma$ will always denote a group isomorphic to $\mathbb{Z}_{p}$.

\subsection{A little group theory.}

Definition. Recall that a group $G$ is finite-by-nilpotent if it has a finite normal subgroup $N$ such that $G / N$ is nilpotent.

Lemma. Suppose that $G$ is a compact p-adic Lie group that is finite-by-nilpotent and has no elements of order $p$.

(1) $G$ has a maximal finite normal subgroup $\Delta^{+}(G)$;

(2) $G / \Delta^{+}$is a nilpotent pro-p group without elements of order $p$.

Proof. For part (1) see [3, 1.3]. Part (2) follows from [1, Lemma 4.1].

2.3. Properties of $\mathbb{Z}_{p} G$. We record some standard properties of Iwasawa algebras that we will use without further comment; see [2] for references to proofs.

Lemma. Suppose that $G$ is a compact p-adic Lie group of dimension d.

(1) $\mathbb{Z}_{p} G$ is a left and right Noetherian ring;

(2) $\mathbb{Z}_{p} G$ is an Auslander-Gorenstein ring of dimension $d+1$;

(3) $\mathbb{Z}_{p} G$ has finite global dimension if and only if $G$ has no elements of order $p$. 
2.4. The canonical dimension function. Recall that if $R$ is an AuslanderGorenstein ring, then there is a canonical dimension function $\delta$ on the category of non-zero finitely generated $R$-modules, given by $\delta(M)=\operatorname{dim} R-j_{R}(M)$ where $j_{R}(M)=\inf \left\{j \mid \operatorname{Ext}_{R}^{j}(M, R) \neq 0\right\}$.

We say that a module $M$ is pseudo-null if $\delta(M) \leqslant \operatorname{inj} \cdot \operatorname{dim}(R)-2$.

When $R=\mathbb{Z}_{p} G$ for $G$ a compact $p$-adic Lie group, we write $d_{G}(M)$ for $\delta(M)$. Then $M$ is pseudo-null as a $\mathbb{Z}_{p} G$-module when $d_{G}(M) \leqslant \operatorname{dim} G-1$.

\subsection{Homology and base change.}

Lemma. Suppose we have rings $R$ and $S$, a ring homomorphism $R \rightarrow S$, a right $S$-module $N$, and a left $R$-module $M$.

(1) If $S$ is flat as a right $R$-module, then

$$
\operatorname{Tor}_{i}^{R}(N, M) \cong \operatorname{Tor}_{i}^{S}\left(N, S \otimes_{R} M\right)
$$

for each $i \geqslant 0$.

(2) In general, there is a base change spectral sequence

$$
E_{i j}^{2}=\operatorname{Tor}_{i}^{S}\left(N, \operatorname{Tor}_{j}^{R}(S, M)\right) \Longrightarrow \operatorname{Tor}_{i+j}^{R}(N, M) .
$$

Proof. Part (1) follows immediately from part (2). Part (2) is [15, Theorem 5.6.6].

\subsection{Computation of homology groups when $G \cong \mathbb{Z}_{p}$.}

Lemma. If $G=\mathbb{Z}_{p}=\langle z\rangle$ and $M$ is a profinite $\mathbb{Z}_{p} G$-module, then

(1) $H_{i}(G, M)=0$ unless $i=0,1$;

(2) $H_{0}(G, M)=M /(z-1) M$;

(3) $H_{1}(G, M)=M^{G}=\operatorname{ker}(z-1): M \rightarrow M$.

Proof. The map $\mathbb{Z}_{p} G \rightarrow \mathbb{Z}_{p} G$ sending $\alpha$ to $(z-1) \alpha$ defines a projective resolution of $\mathbb{Z}_{p}$ as a $\mathbb{Z}_{p} G$-module. All three parts follow easily.

2.7. Localisation at augmentation ideals. Recall that a semiprime ideal $I$ in a ring $R$ is localisable if the set of elements of $r$ in $R$ such that $r+I$ is regular in $R / I$ forms an Ore set in $R$. The following result of Ardakov explains, at least in part, the importance of the condition that $G$ be finite-by-nilpotent.

Theorem (Theorem A of [1]). If $G$ is a compact $p$-adic Lie group and $H$ is a closed normal subgroup, then the kernel $I_{H, G}$ of the augmentation map $\mathbb{Z}_{p} G \rightarrow \mathbb{Z}_{p} G / H$ is localisable if and only if $H$ is a finite-by-nilpotent group.

2.8. Akashi series. The material in this section is largely taken from [8, section 4].

Definition. If $M$ is a finitely generated torsion $\mathbb{Z}_{p} \Gamma$-module, then there is an exact sequence of $\mathbb{Z}_{p} \Gamma$-modules

$$
0 \rightarrow \bigoplus_{i=1}^{r} \mathbb{Z}_{p} \Gamma / \mathbb{Z}_{p} \Gamma f_{i} \rightarrow M \rightarrow D \rightarrow 0
$$

with $D$ pseudo-null. The characteristic element of $M$ is defined by

$$
f_{M}=\prod f_{i}
$$

and is uniquely determined up to multiplication by a unit in $\mathbb{Z}_{p} \Gamma$.

Now write $Q(\Gamma)$ for the field of fractions of $\mathbb{Z}_{p} \Gamma$. 
Definition. If $G$ is isomorphic to a semidirect product $G \cong H \rtimes \Gamma$ and $M$ is a finitely generated $\mathbb{Z}_{p} G$-module such that $H_{j}(H, M)$ is a torsion $\mathbb{Z}_{p} \Gamma$-module for each $j \geq 0$, then the Akashi series of $M$ is given by

$$
A k_{H}(M)=\prod_{j \geqslant 0}\left(f_{H_{j}(H, M)}\right)^{(-1)^{j}} \in Q(\Gamma) /\left(\mathbb{Z}_{p} \Gamma\right)^{\times} .
$$

In this case we say that the Akashi series of $M$ is well-defined. We will suppress the subscript $H$ when no confusion will result.

Remark. Strictly speaking, our definition of the Akashi series is a little more general than that in [8]; we extend their definition to some modules that need not be finitely generated over $\mathbb{Z}_{p} H$. One consequence of this is that unlike the version in [8], if a $p$-torsion module has well-defined Akashi series, it need not be trivial. However, the proofs of the lemma below are identical.

Lemma. Suppose $G$ is isomorphic to a semidirect product $G \cong H \rtimes \Gamma$.

(1) If $0 \rightarrow L \rightarrow M \rightarrow N \rightarrow 0$ is a short exact sequence of finitely generated $\mathbb{Z}_{p} G$-modules with well-defined Akashi series, then $A k(M)=A k(L) \cdot A k(N)$.

(2) If $M$ is a finitely generated $\mathbb{Z}_{p} G$-module with well-defined Euler characteristic, then $M$ has well-defined Akashi series and

$$
\chi(G, M)=|\epsilon(A k(M))|_{p}^{-1}
$$

where $\epsilon$ is the augmentation map $\mathbb{Z}_{p} \Gamma \rightarrow \mathbb{Z}_{p}$.

\section{Characterisation of When the nature of the Zeroth HOMOLOGY GROUP SUFFICES TO DETERMINE WELL-DEFINITION}

3.1. Modules with well-defined Euler characteristic. Suppose that $G$ is any compact $p$-adic Lie group with no elements of order $p$. We want to study those finitely generated left $\mathbb{Z}_{p} G$-modules $M$ with well-defined Euler characteristic. Since the groups $H_{i}(G, M) \cong \operatorname{Tor}_{i}^{\mathbb{Z}_{p} G}\left(\mathbb{Z}_{p}, M\right)$ are finitely generated $\mathbb{Z}_{p}$-modules, we just need to know when they are all $p$-torsion.

Theorem. If $G$ is a compact p-adic Lie group without elements of order $p$, then $G$ is finite-by-nilpotent if and only the following are equivalent for a finitely generated left $\mathbb{Z}_{p} G$-module $M$ :

(1) $M$ has well-defined Euler characteristic;

(2) $H_{0}(G, M)$ is finite.

Proof. Using the Theorem in $\$ 2.7$, we know that $G$ is finite-by-nilpotent if and only if $S:=\mathbb{Z}_{p} G \backslash I_{G, G}$ is an Ore set.

Suppose first that $S$ is an Ore set so that we may form the localisation $\mathbb{Z}_{p} G_{S}$. Then $\mathbb{Z}_{p} G_{S}$ is a local ring with maximal ideal $\left(I_{G, G}\right)_{S}$ and residue field $\mathbb{Q}_{p}$.

Since $\mathbb{Z}_{p}$ is a $\mathbb{Z}_{p} G$-bimodule, $\mathbb{Z}_{p} \otimes_{\mathbb{Z}_{p} G} M$ is a left $\mathbb{Z}_{p} G$-module. As we also have $\left(\mathbb{Z}_{p} \otimes_{\mathbb{Z}_{p} G} P\right)_{S} \cong \mathbb{Q}_{p} \otimes_{\mathbb{Z}_{p} G_{S}} P_{S}$ for every finitely generated projective left $\mathbb{Z}_{p} G$-module $P$, it follows that

$$
\left(\operatorname{Tor}_{i}^{\mathbb{Z}_{p} G}\left(\mathbb{Z}_{p}, M\right)\right)_{S} \cong \operatorname{Tor}_{i}^{\mathbb{Z}_{p} G_{S}}\left(\mathbb{Q}_{p}, M_{S}\right) \text { for each } i \geqslant 0 .
$$

Moreover, since $G$ acts trivially on each group $\operatorname{Tor}_{i}^{\mathbb{Z}_{p} G}\left(\mathbb{Z}_{p}, M\right)$, these groups are all $p$-torsion if and only if they are all $S$-torsion, and this is true if and only if $\operatorname{Tor}_{i}^{\mathbb{Z}_{p} G_{S}}\left(\mathbb{Q}_{p}, M_{S}\right)=0$ for every $i \geqslant 0$. 
As $\mathbb{Z}_{p} G_{S}$ is local with residue field $\mathbb{Q}_{p}$ and $M_{S}$ is finitely generated over $\mathbb{Z}_{p} G_{S}$, Nakayama's Lemma tells us that $\mathbb{Q}_{p} \otimes_{\mathbb{Z}_{p} G_{S}} M_{S}=0$ if and only if $M_{S}=0$. Thus the Euler characteristic of $M$ is well-defined if and only if $M_{S}=0$ if and only if $\mathbb{Z}_{p} \otimes_{\mathbb{Z}_{p} G} M$ is finite as required.

Suppose now that $S$ is not an Ore set. This means that we can find $r \in \mathbb{Z}_{p} G$ and $s \in S$ such that $S r \cap \mathbb{Z}_{p} G s=\emptyset$. We consider the cyclic left $\mathbb{Z}_{p} G$-module $M=\mathbb{Z}_{p} G / \mathbb{Z}_{p} G\langle r, s\rangle$ for this pair $r, s$.

There is a free resolution of $M$ that begins as follows:

$$
\cdots \rightarrow\left(\mathbb{Z}_{p} G\right)^{d} \stackrel{d_{1}}{\rightarrow}\left(\mathbb{Z}_{p} G\right)^{2} \stackrel{d_{0}}{\rightarrow} \mathbb{Z}_{p} G \rightarrow M \rightarrow 0,
$$

with $d_{0}(\alpha, \beta)=\alpha r+\beta s$.

Now applying $\mathbb{Z}_{p} \otimes_{\mathbb{Z}_{p} G}(-)$ to this resolution yields a complex

$$
\mathbb{Z}_{p}^{d} \stackrel{\overline{d_{1}}}{\rightarrow} \mathbb{Z}_{p}^{2} \stackrel{\overline{d_{0}}}{\rightarrow} \mathbb{Z}_{p} \rightarrow 0
$$

with homology $H_{\bullet}(G, M)$.

The condition that $S r \cap \mathbb{Z}_{p} G s=\emptyset$ means that if $d_{0}(\alpha, \beta)=0$, then $\alpha \in I_{G, G}$. Since $S$ is multiplicatively closed it follows also that $\beta \in I_{G, G}$. Thus

and so $\overline{d_{1}}=0$.

$$
\operatorname{Im}\left(d_{1}\right)=\operatorname{ker}\left(d_{0}\right) \subseteq I_{G, G}\left(\mathbb{Z}_{p} G\right)^{2}
$$

We also have $\overline{d_{0}}(a, b)=a \epsilon(r)+b \epsilon(s)$ where $\epsilon$ is the augmentation map $\mathbb{Z}_{p} G \rightarrow \mathbb{Z}_{p}$. As $s \in S, \epsilon(s) \neq 0$ and so $\overline{d_{0}}$ is not the zero map. It follows that $H_{0}(G, M)$ is finite and $H_{1}(G, M) \cong \mathbb{Z}_{p}$.

3.2. Multiplicativity of the Euler characteristic. Because the $S$-torsion modules form an abelian subcategory of all finitely generated $\mathbb{Z}_{p} G$-modules, we may prove the following:

Proposition. Suppose that $G$ is a finite-by-nilpotent compact p-adic Lie group. If $0 \rightarrow L \rightarrow M \rightarrow N \rightarrow 0$ is a short exact sequence of finitely generated left $\mathbb{Z}_{p} G$ modules, then $M$ has well-defined Euler characteristic if and only if both $L$ and $N$ have well-defined Euler characteristic. Moreover, in this case,

$$
\chi(G, M)=\chi(G, L) \cdot \chi(G, N) .
$$

Proof. The first part follows from the Theorem in $₫ 3.1$. The second part can be read off from the long exact sequence of homology.

3.3. Modules with well-defined Akashi series. There are analogous results for Akashi series, in particular the following theorem:

Theorem. Suppose $G$ is isomorphic to a semidirect product $H \rtimes \Gamma$, and define

$$
T:=\mathbb{Z}_{p} G \backslash \operatorname{ker}\left(\mathbb{Z}_{p} G \rightarrow \mathbb{Z}_{p} \Gamma\right) .
$$

The Akashi series of $M$ is well-defined for every finitely generated left $\mathbb{Z}_{p} G$-module $M$ such that $H_{0}(H, M)$ is a torsion $\mathbb{Z}_{p} \Gamma$-module if and only if $T$ is an Ore set in $\mathbb{Z}_{p} G$ if and only if $H$ is finite-by-nilpotent.

Proof. The proof is nearly identical to that of the Theorem in $\$ 3.1$, so we describe it briefly:

Once again, that $T$ is an Ore set if and only if $H$ is finite-by-nilpotent follows immediately from the Theorem in $\$ 2.7$. 
If $T$ is an Ore set, then $\mathbb{Z}_{p} G_{T}$ is a local ring with maximal ideal $\left(I_{H, G}\right)_{T}$ and $H_{0}(H, M)$ is a torsion $\mathbb{Z}_{p} \Gamma$-module if and only if

$$
H_{0}(H, M)_{T} \cong M \otimes_{\mathbb{Z}_{p} G_{T}} \mathbb{Z}_{p} G_{T} /\left(I_{H, G}\right)_{T}=0
$$

if and only if $M_{T}=0$. Thus $H_{j}(H, M)_{T}=0$ for every $j \geq 0$ if and only if $H_{0}(H, M)_{T}=0$, and the former holds if and only if $H_{0}(H, M)$ is $\mathbb{Z}_{p} \Gamma$-torsion.

Conversely, if $T$ is not an Ore set, then there are elements $r \in \mathbb{Z}_{p} G$ and $t \in T$ such that there are no elements $r^{\prime} \in \mathbb{Z}_{p} G$ and $t^{\prime} \in T$ with $r^{\prime} t=t^{\prime} r$. Thus the kernel of the map

$$
\left(\mathbb{Z}_{p} G\right)^{2} \rightarrow \mathbb{Z}_{p} G ; \quad(\alpha, \beta) \mapsto \alpha r+\beta t
$$

is contained in $\left(I_{H, G}\right)^{2}$. Using this fact to compute the homology groups $H_{j}(H, M)$ for $M=\mathbb{Z}_{p} G / \mathbb{Z}_{p} G\langle r, t\rangle$, we obtain that $H_{0}(H, M)$ is $\mathbb{Z}_{p} \Gamma$-torsion but $H_{1}(H, M)$ is not $\mathbb{Z}_{p} \Gamma$-torsion.

Corollary. If $G$ is as above and $0 \rightarrow L \rightarrow M \rightarrow N \rightarrow 0$ is a short exact sequence of finitely generated $\mathbb{Z}_{p} G$-modules, then $M$ has well-defined Akashi series if and only if $L$ and $N$ both have well-defined Akashi series.

\section{Triviality of the Euler characterstid FOR PSEUDO-NULL MODULES}

4.1. Reduction to torsion-free nilpotent $G$. Our main goal now is to prove the second part of our main result: that if $G$ is a finite-by-nilpotent compact $p$-adic Lie group without $p$-torsion and $M$ is a finitely generated pseudo-null $\mathbb{Z}_{p} G$-module with well-defined Euler characteristic, then $\chi(G, M)=1$.

In fact we prove the apparently stronger result that if $G$ is a finite-by-nilpotent compact $p$-adic Lie group and $H$ is a closed normal subgroup such that $G \cong H \rtimes \Gamma$, then whenever $M$ is a finitely generated pseudo-null $\mathbb{Z}_{p} G$-module with well-defined Akashi series we have $A k_{H}(M)=1$. To see that the Euler characteristic version follows from this, observe that (except in the trivial case where $G$ is finite) we can always find such a closed normal subgroup $H$ and apply part (2) of the Lemma in 2.8

We first reduce to the case where $G$ is nilpotent and pro- $p$.

Lemma. Suppose that $G \cong H \rtimes \Gamma$ is a compact p-adic Lie group with finite normal subgroup $\Delta$ such that $(|\Delta|, p)=1$. If $M$ is a finitely generated $\mathbb{Z}_{p} G$-module, then

$$
H_{i}(H, M) \cong H_{i}\left(H / \Delta, M_{\Delta}\right) \text { for each } i \geqslant 0
$$

as $\mathbb{Z}_{p} \Gamma$-modules, and so $A k_{H}(M)=A k_{H / \Delta}\left(M_{\Delta}\right)$ if either is well-defined; of course $G / \Delta \cong(H / \Delta) \rtimes \Gamma$.

Moreover, $d_{G / \Delta}\left(M_{\Delta}\right) \leqslant d_{G}(M)$, and so $M_{\Delta}$ is a pseudo-null $\mathbb{Z}_{p} G / \Delta$-module if $M$ is a pseudo-null $\mathbb{Z}_{p} G$-module.

Proof. First recall that $\mathbb{Z}_{p}$ with the trivial $\Delta$-action is a projective right $\mathbb{Z}_{p} \Delta$ module as $|\Delta|$ is a unit in $\mathbb{Z}_{p}$. Since the induction functor $(-) \hat{\otimes}_{\mathbb{Z}_{p} \Delta} \mathbb{Z}_{p} H$ from profinite right $\mathbb{Z}_{p} \Delta$-modules to profinite right $\mathbb{Z}_{p} H$-modules is left-adjoint to the restriction functor, it sends projective modules to projective modules, and so in particular $\mathbb{Z}_{p} H / \Delta \cong \mathbb{Z}_{p} \hat{\otimes}_{\mathbb{Z}_{p} \Delta} \mathbb{Z}_{p} H$ is a projective profinite right $\mathbb{Z}_{p} H$-module and hence flat as a right $\mathbb{Z}_{p} H$-module.

Using part (1) of the Lemma in 2.5 we may conclude that $H_{i}(H, M) \cong$ $H_{i}\left(H / \Delta, H_{0}(\Delta, M)\right)$ for each $i \geqslant 0$ as $\mathbb{Z}_{p} \Gamma$-modules, and the first part follows. 
By considering a finitely generated projective resolution of $M$ we can also show that

$$
\operatorname{Ext}_{\mathbb{Z}_{p} G}^{j}\left(M, \mathbb{Z}_{p} G\right) \otimes_{\mathbb{Z}_{p} \Delta} \mathbb{Z}_{p} \cong \operatorname{Ext}_{\mathbb{Z}_{p} G / \Delta}^{j}\left(M_{\Delta}, \mathbb{Z}_{p} G / \Delta\right)
$$

for each $j \geqslant 0$, and the second part follows too as $\operatorname{dim} G=\operatorname{dim} G / \Delta$.

By applying this Lemma in the case where $\Delta=\Delta^{+}$is the maximal finite normal subgroup of $G$ and using Lemma 2.2. we have reduced the calculation of Euler characteristics of finitely generated pseudo-null $\mathbb{Z}_{p} G$-modules where $G$ is finite-bynilpotent without elements of order $p$ to the case where $G$ is torsion-free nilpotent and pro- $p$.

4.2. The torsion-free nilpotent case. Suppose now that $G$ is a finitely generated nilpotent pro- $p$ group without torsion and we have a fixed decomposition $G \cong H \rtimes \Gamma$.

Lemma. Suppose that $M$ is a $\mathbb{Z}_{p} G$-module with well-defined Akashi series and there exists $z \in Z(G) \cap H$ such that $Z=\langle z\rangle$ is an isolated subgroup of $G$ acting trivially on $M$. Then $A k_{H}(M)=1$.

Proof. First notice that $H_{0}(H, M)=H_{0}(H / Z, M)$ is a torsion $\mathbb{Z}_{p} \Gamma$-module, that $G / Z \cong H / Z \rtimes \Gamma$ and that $H / Z$ is also torsion-free and nilpotent so that $A k_{H / Z}(M)$ is well-defined by the Theorem in $\$ 3.3$ Now the proof is nearly identical to the proof of [5, Corollary 12.2], but we'll sketch it here for the sake of the reader.

By the Lemma in 2.6. $H_{i}(Z, M)$ vanishes for $i>1$ and is isomorphic to $M$ as a left $\mathbb{Z}_{p} G / Z$-module for $i=0,1$ since $Z$ is central in $G$. Thus part (2) of the Lemma in 2.5 describes a spectral sequence of $\mathbb{Z}_{p} \Gamma$-modules with second page

$$
E_{i j}=H_{i}\left(H / Z, H_{j}(Z, M)\right)
$$

that is concentrated in rows $j=0,1$. By [15, Exercise 5.2.2], for example, this yields a long exact sequence

$$
\cdots \rightarrow H_{n+1}(H, M) \rightarrow H_{n+1}(H / Z, M) \rightarrow H_{n-1}(H / Z, M) \rightarrow H_{n}(H, M) \rightarrow \cdots
$$

of $\mathbb{Z}_{p} \Gamma$-modules.

The result now follows from the multiplicativity of characteristic elements.

Theorem. If $G \cong H \rtimes \Gamma$ is a finite-by-nilpotent $p$-adic Lie group without elements of order $p$ and $M$ is a pseudo-null $\mathbb{Z}_{p} G$-module with well-defined Akashi series, then $A k_{H}(M)=1$.

Proof. By applying the reduction in section 4.1 we may assume that $G$ is nilpotent and torsion-free.

Since $M$ is Noetherian and $A k_{H}(0)=1$, we may define $N$ to be a maximal submodule of $M$ such that $A k_{H}(N)=1$. Using part (1) of the Lemma in $\$ 2.8$ and the Corollary in 33.3 , we see that every non-zero submodule $L$ of $M / N$ satisfies $A k_{H}(L) \neq 1$. Thus after replacing $M$ by $M / N$ it suffices to prove that $M$ must have a non-zero submodule $L$ with $A k_{H}(L)=1$.

Next recall $([10,4.5])$ that whenever we have a finitely generated module $M$ over a Auslander-Gorenstein ring, it has a critical submodule, i.e. a submodule $N$ with the property that every proper quotient has strictly smaller canonical dimension. Using this fact and the remarks in the first paragraph we may assume that our module $M$ is critical. 
Now pick an isolated one-dimensional subgroup $Z$ of $Z(G) \cap H$; then $M^{Z}$ is a $\mathbb{Z}_{p} G$-submodule of $M$. By the Lemma above, $A k_{H}\left(M^{Z}\right)=1$ and so we may assume (by part (3) of the Lemma in $\$ 2.6$ ) that $H^{1}(Z, M)=M^{Z}=0$.

Now the homology spectral sequence

$$
E_{i j}^{2}=H_{i}\left(H / Z, H_{j}(Z, M)\right) \Longrightarrow H_{i+j}(H, M)
$$

has only one non-trivial row on the second page and so $H_{i}(H, M)=H_{i}\left(H / Z, M_{Z}\right)$ as $\mathbb{Z}_{p} \Gamma$-modules. Thus $A k_{H}(M)=A k_{H / Z}\left(M_{Z}\right)$.

But $d_{G}\left(M_{Z}\right)<d_{G}(M)$, since $M$ is critical. Also $d_{G}\left(M_{Z}\right)=d_{G / Z}\left(M_{Z}\right)$ by the Rees Lemma ([12, Theorem 9.37], for example). It follows that $M_{Z}$ is a pseudonull $\mathbb{Z}_{p} G / Z$-module with well-defined Akashi series, and by induction on $\operatorname{dim}(G)$ we have $A k_{H / Z}\left(M_{Z}\right)=1$; the result is trivial when $H=1$ by the definition of characteristic element.

Corollary. If $G$ is a finite-by-nilpotent p-adic Lie group without elements of order $p$ and $M$ is a pseudo-null $\mathbb{Z}_{p} G$-module with well-defined Euler characterstic, then $\chi(G, M)=1$.

4.3. Partial converses. We now discuss some results that put restrictions on the set of groups for which the second conclusion of the main theorem holds.

Definition. We say that pseudo-nulls are $\chi$-trivial for $G$ if whenever a finitely generated pseudo-null left $\mathbb{Z}_{p} G$-module $M$ has well-defined Euler characteristic we have $\chi(G, M)=1$.

Theorem. Suppose that $G$ is a compact p-adic Lie group without p-torsion and pseudo-nulls are $\chi$-trivial for $G$. Then

(1) pseudo-nulls are $\chi$-trivial for every closed subgroup $H$ of $G$;

(2) $G$ is p-nilpotent, i.e. $G / \Delta^{+}(G)$ is pro-p;

(3) $\operatorname{dim} C_{G}(g)>1$ for all $g \in G$ or $G$ has dimension 1 ;

(4) if $G$ is isomorphic to a semidirect product $\mathbb{Z}_{p}^{d} \rtimes \mathbb{Z}_{p}$, then $G$ is nilpotent;

(5) if $G$ is split-reductive, then it is abelian.

Proof. For part (1), suppose $H$ is a closed subgroup of $G$ and $N$ is any finitely generated left $\mathbb{Z}_{p} H$-module. By Shapiro's Lemma (see [11, Theorem 6.10.9], for example) we have $H_{i}\left(G, \mathbb{Z}_{p} G \otimes_{\mathbb{Z}_{p} H} N\right) \cong H_{i}(H, N)$ for each $i \geqslant 0$, so it suffices to prove that if $N$ is pseudo-null as a $\mathbb{Z}_{p} H$-module, then $\mathbb{Z}_{p} G \otimes_{\mathbb{Z}_{p} H} N$ is pseudo-null as a $\mathbb{Z}_{p} G$-module.

By inducing a finitely generated projective resolution of $N$ as a $\mathbb{Z}_{p} H$-module to a projective resolution of $\mathbb{Z}_{p} G \otimes_{\mathbb{Z}_{p} H} N$ as a $\mathbb{Z}_{p} G$-module, we see that

$$
\operatorname{Ext}_{\mathbb{Z}_{p} H}^{j}\left(N, \mathbb{Z}_{p} H\right) \otimes_{\mathbb{Z}_{p} H} \mathbb{Z}_{p} G \cong \operatorname{Ext}_{\mathbb{Z}_{p} G}^{j}\left(\mathbb{Z}_{p} G \otimes_{\mathbb{Z}_{p} H} N, \mathbb{Z}_{p} G\right)
$$

for each $j \geqslant 0$ and we are done.

Part (2) follows from [4, Lemma $7.4 \&$ Theorem 11.5]. In particular there is a pseudo-null $p$-torsion $\mathbb{Z}_{p} G$-module $M$ with $\chi(G, M) \neq 1$.

For part (3), since all open subgroups are closed, part (1) tells us that it suffices to prove the result for an open subgroup of $G$. The result now follows immediately from Totaro's Theorem quoted in the introduction.

For part (4), suppose $G \cong \mathbb{Z}_{p}^{d} \rtimes \mathbb{Z}_{p}$, let $H$ be the closed normal subgroup of $G$ isomorphic to $\mathbb{Z}_{p}^{d}$ and let $g$ generate a complement to $H$ in $G$. Now the action of $g$ on $H$ has a minimal polynomial $p(t)$, say, and we may write $p(t)=(t-1)^{r} q(t)$ with 
$q(t)$ and $(t-1)$ relatively prime. If $q$ is constant, then $G$ is nilpotent, so we may assume it is not. Let $K=\langle\operatorname{ker} q(g), g\rangle$, a closed subgroup $G$ with $\operatorname{dim} C_{K}(g)=1$. By part (3) pseudo-nulls are not $\chi$-trivial for $K$, and the result follows from part (2).

To prove part (5) we first notice that parts (1) and (4) imply that it suffices to show that if $G$ is not abelian, then it must have a non-nilpotent closed subgroup isomorphic to a semidirect product $\mathbb{Z}_{p}^{d} \rtimes \mathbb{Z}_{p}$. If the associated Lie algebra is splitreductive but not abelian, then $G$ has a non-abelian subgroup isomorphic to a semi-direct product $\mathbb{Z}_{p} \rtimes \mathbb{Z}_{p}$.

Remarks.

(1) It is easy to see that the proof of part (5) applies to a much wider class of groups than non-abelian split-reductive groups.

(2) However, one class of examples that such arguments don't easily apply to includes groups that are four-dimensional and soluble and which are isomorphic to a semidirect product $H_{3} \rtimes \mathbb{Z}_{p}$ where $H_{3}$ is a three-dimensional Heisenberg pro- $p$ group and the complementary copy of $\mathbb{Z}_{p}$ is generated by an element that induces an automorphism of infinite order on $H_{3}$ that acts trivially on the centre of $H_{3}$ and fixes no other one-dimensional subgroup.

\section{ACKNOWLEDGMENTS}

Much of this work was done whilst the author was a EPSRC postdoctoral fellow under research grant EP/C527348/1. He would like to thank Konstantin Ardakov for many helpful conversations.

\section{REFERENCES}

[1] Konstantin Ardakov. Localisation at augmentation ideals in Iwasawa algebras. Glasg. Math. $J ., 48(2): 251-267,2006$. MR 2256976 (2007h:16030)

[2] K. Ardakov and K. A. Brown. Ring-theoretic properties of Iwasawa algebras: a survey. Doc. Math., Extra Vol.:7-33, 2006. MR.2290583 (2007k:11185)

[3] Konstantin Ardakov and Kenneth A. Brown. Primeness, semiprimeness and localisation in Iwasawa algebras. Trans. Amer. Math. Soc., 359(4):1499-1515 (electronic), 2007. MR.2272136 (2007j:16031)

[4] Konstantin Ardakov and Simon Wadsley. Characteristic elements for $p$-torsion Iwasawa modules. J. Algebraic Geom., 15(2):339-377, 2006. MR2199061 (2006m:11152)

[5] Konstantin Ardakov and Simon Wadsley. $K_{0}$ and the dimension filtration for $p$-torsion Iwasawa modules. Proc. Lond. Math. Soc. (3), 97(1):31-59, 2008. MR2434090 (2010b:11147)

[6] John Coates. Fragments of the $\mathrm{GL}_{2}$ Iwasawa theory of elliptic curves without complex multiplication. In Arithmetic theory of elliptic curves (Cetraro, 1997), volume 1716 of Lecture Notes in Math., pages 1-50. Springer, Berlin, 1999. MR1754685 (2002c:11060)

[7] John Coates, Takako Fukaya, Kazuya Kato, Ramdorai Sujatha, and Otmar Venjakob. The $\mathrm{GL}_{2}$ main conjecture for elliptic curves without complex multiplication. Publ. Math. Inst. Hautes Études Sci., 101:163-208, 2005. MR2217048(2007b:11172)

[8] John Coates, Peter Schneider, and Ramdorai Sujatha. Links between cyclotomic and GL 2 Iwasawa theory. Doc. Math., Extra Vol. (Kazuya Kato's fiftieth birthday): 187-215 (electronic), 2003. MR2046599 (2005c:11134)

[9] John Coates and Ramdorai Sujatha. Euler-Poincaré characteristics of abelian varieties. C. $R$. Acad. Sci. Paris Sér. I Math., 329(4):309-313, 1999. MR1713337 (2001g:11090)

[10] Thierry Levasseur. Some properties of noncommutative regular graded rings. Glasg. Math. J., 34(3):277-300, 1992. MR.1181768 (93k:16045)

[11] Luis Ribes and Pavel Zalesskii. Profinite groups, volume 40 of Ergebnisse der Mathematik und ihrer Grenzgebiete. 3. Folge. A Series of Modern Surveys in Mathematics. Springer-Verlag, Berlin, 2000. MR 1775104 (2001k:20060) 
[12] Joseph J. Rotman. An introduction to homological algebra, volume 85 of Pure and Applied Mathematics. Academic Press Inc. [Harcourt Brace Jovanovich Publishers], New York, 1979. MR.538169 (80k:18001)

[13] Jean-Pierre Serre. La distribution d'Euler-Poincaré d'un groupe profini. In Galois representations in arithmetic algebraic geometry (Durham, 1996), volume 254 of London Math. Soc. Lecture Note Ser., pages 461-493. Cambridge University Press, Cambridge, 1998. MR.1696505 (2000g:22017)

[14] Burt Totaro. Euler characteristics for p-adic Lie groups. Inst. Hautes Études Sci. Publ. Math., 90:169-225, 1999. MR 1813226 (2002f:22032)

[15] Charles A. Weibel. An introduction to homological algebra, volume 38 of Cambridge Studies in Advanced Mathematics. Cambridge University Press, Cambridge, 1994. MR.1269324 (95f:18001)

Homerton College, University of Cambridge, Cambridge, CB2 8PQ, United Kingdom E-mail address: S.J.Wadsley@dpmms.cam.ac.uk 